\title{
LA CÉROÏDE-LIPOFUSCINOSE DE
} L'AMERICAN STAFFORDSHIRE TERRIER

\author{
NEURONAL CEROID LIPOFUSCINOSIS IN \\ AMERICAN STAFFORDSHIRE TERRIERS
}

Par Marie ABITBOL ${ }^{1}$

(Communication présentée le 7 auril 2011)

\begin{abstract}
RÉSUMÉ
Les céroïdes-lipofuscinoses neuronales (CLNs) humaines constituent un groupe d'encéphalopathies progressives et héréditaires caractérisées par une perte progressive de la vue, une dégradation des fonctions motrices et mentales, des crises épileptiformes et un décès prématuré. Les chiens de race American Staffordshire Terrier (AST) présentent une forme adulte et autosomique récessive de CLN qui se traduit par une ataxie statique et dynamique incurable, nécessitant l'euthanasie de l'animal à un stade avancé de la maladie. Grâce à une étude d'association et de liaison menée chez un grand nombre d'AST, une mutation dans le gène de l'arylsulfatase $\mathrm{G}$ a été découverte chez les chiens atteints. Elle provoque le remplacement d'un acide aminé dans la protéine et conduit à une baisse d'activité de l'activité arylsulfatase de $75 \%$ dans les leucocytes des chiens atteints. Ces travaux ont permis de mettre en place un test de dépistage de la maladie chez l'AST, de révéler l'importance de l'arylsulfatase $\mathrm{G}$ dans l'homéostasie neuronale et d'identifier une famille de gènes candidats pour les formes adultes de CLN humaines.
\end{abstract}

Mots-clés : chien, héréditaire, ADN, neurone, céroïde-lipofuscinose, ataxie, cervelet.

\section{SUMMARY}

Neuronal ceroid lipofuscinoses (NCLs) represent a group of inherited progressive encephalopathies characterized by progressive loss of vision, mental or motor deterioration, epileptic seizures and premature death. A spontaneous canine model of adult American Staffordshire Terriers developing an autosomal recessive NCL has been described. Through a combined genome-wide association and linkage study, a worldwide breed-specific variant in the arylsulfatase G (ARSG) gene was identified. ARSG encodes a lysosomal sulfatase. The variant causes a non-conservative amino-acid substitution in the protein. The missense substitution leads to a $75 \%$ sulfatase activity decrease in leucocytes from affected dogs. These results unravel the key role played by ARSG in NCL and neuronal homeostasis and suggest that sulfatase activity might be evaluated in human patients affected by late-onset forms of NCL. Further, a canine genomic predictive DNA-test has been developed to serve the future of the breed.

Key words: dog, inherited disease, DNA, neuron, lipofuscinosis, ataxia, cerebellum.

(1) UMR955 INRA-ENVA Génétique fonctionnelle et médicale, École Nationale Vétérinaire 7, Avenue du Général de Gaulle 94704 Maisons-Alfort. 


\section{LES CÉROÏDES-LIPOFUSCINOSES NEURONALES (CLNS)}

\section{Classification des CLNs}

Chez l'homme, les CLNs constituent un groupe de maladies neurodégénératives caractérisées par diverses altérations neurologiques et l'accumulation intracellulaire d'une substance auto-fluorescente. Elles représentent le groupe le plus fréquent d'encéphalopathies chez les enfants (Jalanko \& Braulke, 2009). Dix pour cent d'entre elles sont des formes sporadiques ou héréditaires de l'adulte, appelées "maladie de Kufs " (Berkovic et al. 1988 ; Haltia 2006 ; Lewandowska et al. 2009). À l'exception de rares formes adultes autosomiques dominantes, elles ont un mode de transmission autosomique récessif et sont classées en 10 formes génétiques désignées CLN1 à CLN10. Huit formes de CLNs ont été caractérisées au niveau moléculaire : CLN1 à CLN3, CLN5 à CLN8 et CLN10 (Jalanko \& Braulke, 2009). Les gènes responsables de la forme CLN9 et de la maladie de Kufs ou CLN4 ne sont pas encore identifiés.

\section{Physiopathologie des CLNs}

Les CLNs forment un sous-groupe de maladies lysosomiales, caractérisé par une atteinte presque exclusivement neuronale. Les neurones post-mitotiques étant des cellules métaboliquement très actives, le matériel de stockage résultant de l'atteinte lysosomiale ne peut pas être dilué par division des neurones chez les individus atteints de CLN. Le matériel de stockage lysosomial produit des dommages cellulaires et un stress oxydatif responsables de l'apoptose des neurones (Bellettato \& Scarpa, 2010). Si de nombreuses protéines à l'origine des CLNs ont été identifiées, les mécanismes physiopathologiques qui conduisent du dysfonctionnement protéique à la mort neuronale restent largement méconnus. Cependant, il a été montré que la protéine de la forme CLN2 est une enzyme lysosomiale dont les différents substrats n'ont pas été retrouvés dans le matériel de stockage présenté par les patients (Jalanko \& Braulke, 2009). De façon plus générale, de nombreuses protéines CLN lysosomiales et non lysosomiales sont impliquées dans des mécanismes faisant intervenir des compartiments cellulaires autres que les lysosomes (Bellettato \& Scarpa, 2010). De plus, un matériel de stockage auto-fluorescent, comparable à celui identifié dans les CLNs, a été mis en évidence dans des lymphoblastes traités par un agent pharmacologique inhibant l'assemblage des microtubules (Seehafer \& Pearce, 2009). Ainsi, à l'heure actuelle, il est postulé que les trois principaux mécanismes cellulaires impliqués dans la physiopathologie des CLNs sont le trafic intracellulaire et membranaire, l'autophagie et l'homéostasie calcique :

- il a été montré que des protéines associées au cytosquelette étaient anormales dans les neurones atteints des formes CLN1 et CLN5 (von Schantz et al. 2008) et que la protéine de la forme CLN1 (ou protéine CLN1) modulerait les premiers stades de l'endocytose (Saja et al. 2010) ;
- une diminution de l'autophagie, résultant d'une inhibition de la fusion entre autophagosomes et lysosomes a été observée dans la plupart des CLNs (Bellettato \& Scarpa, 2010). En conséquence, des protéines polyubiquitinylées et des mitochondries lésées (sources potentielles de radicaux libres) s'accumulent dans les neurones, le stress du réticulum endoplasmique est activé et ces phénomènes convergent vers l'apoptose du neurone (Zhao \& Ackerman, 2006 ; Bellettato \& Scarpa, 2010);

- un dysfonctionnement lysosomial peut induire la mort cellulaire via une dérégulation de l'homéostasie du calcium. Une concentration élevée de calcium est cytotoxique. Des protéines spécialisées des compartiments mitochondriaux et du réticulum endoplasmique, en particulier, régulent les flux de calcium. Un exemple de perturbation du flux calcique a été rapporté dans les neurones déficients en protéine CLN3 (Luiro et al. 2006).

En conclusion, ces trois mécanismes sont certainement interconnectés : par exemple, il a été mis en évidence que la protéine de la forme CLN5 interagissait avec les protéines des formes CLN1, CLN2, CLN3, CLN6 et CLN8 (Lyly et al. 2009).

\section{LA CLN DE L'AMERICAN STAFFORDSHIRE TERRIER (AST)}

Un grand nombre de modèles animaux de CLNs ont été décrits. La plupart modélisent bien les formes juvéniles et infantiles (www.caninegeneticdiseases.net/CL_site/mainCL.htm et Fiske \& Storts, 1988 ; Jalanko \& Braulke 2009 ; Kawamura et al. 2009).

\section{Une ataxie cérébelleuse chez l'AST}

Chez l'AST, une ataxie cérébelleuse, également appelée abiotrophie cérébelleuse, a été décrite dès 2003, en Europe et aux USA (Hanzlicek et al. 2003 ; Olby et al. 2004). Les chiens atteints présentaient, en début d'évolution de la maladie, une ataxie discrète qui se manifestait surtout lors de changements de direction (angle d'une rue, d'un couloir), lors de la montée ou la descente d'escaliers, de plans inclinés ou encore lors de la nage. Cette apparente maladresse évoluait vers une ataxie permanente de plus en plus marquée. Les chiens présentaient alors une astasie (trouble de l'équilibre au repos, figure 1) et une hypermétrie, surtout marquée sur les membres antérieurs. La montée des escaliers devenait difficile, voire impossible. Certains chiens portaient la tête discrètement inclinée alternativement d'un côté ou de l'autre. Un nystagmus était fréquemment déclenché soit lors d'hyperextension cervicale, soit en faisant rouler le chien sur le dos, et chez certains ASTs, on a observé la présence de tremblements intentionnels de tout le corps. En fin d'évolution, la stabilité du regard était perdue, les chiens devenaient incapables de se mouvoir et de se nourrir seul. Il n'a jamais été mis en évidence de modification comportementale, mise à part une certaine indifférence à l'environne- 


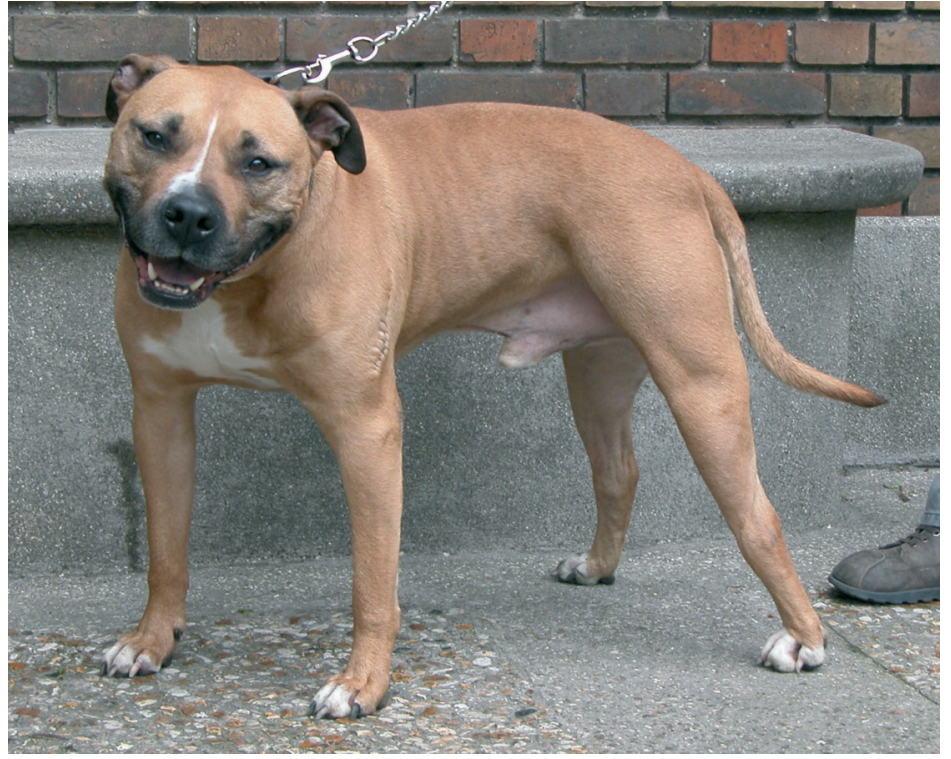

Figure 1 : American Staffordshire Terrier adulte atteint de céroïde-lipofuscinose neuronale. Le polygone de sustentation est augmenté et la tête est discrètement penchée au repos. (Source : laboratoire de Neurobiologie, ENVA).

ment, en fin d'évolution de la maladie. La majorité des chiens atteints a présenté les premiers symptômes de l'ataxie entre les âges de trois à cinq ans (Olby et al. 2004, Abitbol et al. 2010). La maladie évoluait de quelques mois à plusieurs années et une euthanasie de l'animal était généralement pratiquée, à la demande du propriétaire, en fin d'évolution.

\section{Les chiens ataxiques présentent une CLN}

À l'École Nationale Vétérinaire d'Alfort (ENVA), un programme de recherche sur l'ataxie cérébelleuse de l'AST a été mis en œuvre, en 2000, par le Pr Stéphane Blot (laboratoire de Neurobiologie). Une collaboration avec le laboratoire du Dr Natasha Olby, de l'Université de Caroline du Nord, aux USA (Olby et al. 2004), a permis de collecter des informations génétiques et phénotypiques chez 138 ASTs nés en France et aux USA. Soixante dix pour cent des chiens avaient présenté les premiers signes d'ataxie entre trois et cinq ans. Les 58 chiens qui ont été examinés en imagerie par résonnance magnétique ont présenté une réduction de la taille du cervelet et un approfondissement des sillons cérébelleux. L'étude histologique de coupes de cervelet a montré une diminution très importante, voire une disparition d'une catégorie de neurones, les cellules de Purkinje. Des colorations par l'acide périodique Schiff (qui teinte les hydrates de carbone : cérébrosides et glycogène), le noir Soudan (qui colore les lipoprotéines) et le bleu de Luxol (qui colore la myéline et les lipoprotéines), ont mis en évidence la présence de multiples agrégats cytoplasmiques dans les rares cellules de Purkinje restantes (figure 2), dans les neurones du cortex occipital, du noyau de l'hypoglosse, du noyau postérieur du thalamus et dans les cellules pyramidales de l'hippocampe ou corne d'Amon. Ces agrégats ressemblaient fortement à une accumulation de céroïde-lipofuscine. L'analyse, en microscopie électronique, de cellules de Purkinje présentant cette surcharge a permis de mettre en évidence des lysosomes anormaux, contenant des inclusions de matériel dense aux électrons, organisés en bandes linéaires, courbes ou concentriques avec une alternance de bandes sombres et claires (figure 2). La substance blanche, les ganglions lymphatiques, la rate et la rétine ne présentaient aucune anomalie histologiquement visible (Abitbol et al. 2010).
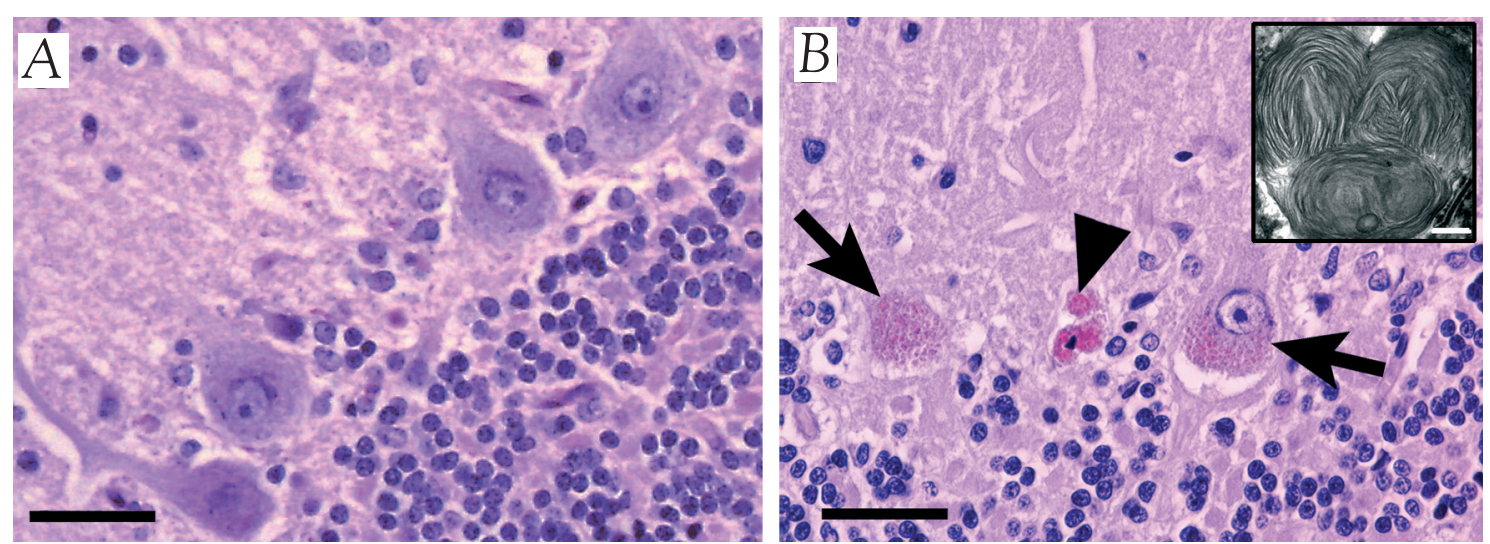

Figure 2 : Coupes transversales de cervelet d'un American Staffordshire Terrier (AST) sain (A) et d'un AST atteint de céroïde-lipofuscinose neuronale (CLN, B), colorées par l'acide périodique Schiff (PAS) et contre-colorées par l'hémalun éosine. (A) Chez le chien indemne de CLN, les cellules de Purkinje PAS négatives sont situées entre la couche granulaire et la couche moléculaire. (B) Chez le chien atteint de CLN, il ne reste que quelques cellules de Purkinje. Les rares cellules de Purkinje (flèches noires) ou débris de Purkinje (triangle noir) présentent une accumulation périnucléaire de matériel PAS positif. (Encart en B) Image en microscopie électronique d'une cellule de Purkinje présentant un lysosome gorgé d'un matériel de stockage organisé en bandes claires et sombres, concentriques ou courbes. Échelles : 50 um et 250nm pour l'encart. (D'après Abitbol et al. 2010). 


\section{Une CLN autosomique récessive d'apparition tardive chez l'AST}

Une étude réalisée en 2004, chez cinq chiens suisses non apparentés atteints d'ataxie cérébelleuse suggéra que l'ataxie pourrait résulter d'une accumulation de matériel de stockage auto-fluorescent et donc être une CLN (Siso et al. 2004)

Les résultats cliniques et histologiques, obtenus à l'aide d'une large cohorte de chiens français et américains, ont permis de confirmer que l'ataxie cérébelleuse de l'AST était en fait une CLN. Le mode de transmission autosomique récessif de cette ataxie, suggéré par l'étude de 2004 (Olby et al. 2004), a été confirmé par l'analyse de 73 AST français pour lesquels une information de pedigree complète était disponible (Abitbol et al. 2010).

Ainsi, les AST américains et français peuvent être atteints d'une CLN d'apparition tardive, qui se transmet sur un mode autosomique récessif. L'AST est le second modèle de CLN héréditaire et tardive caractérisé chez le chien, après le Terrier Tibétain (Farias et al. 2011).

\section{IDENTIFICATION DE LA MUTATION RESPONSABLE DE LA CLN}

\section{Mise en évidence d'un locus associé et lié à la CLN, sur le chromosome 9 canin}

De façon à cartographier le locus responsable de la CLN, 77 chiens français ont été génotypés à l'aide de 247 marqueurs microsatellites répartis de façon homogène sur les chromosomes du chien. La cohorte était composée de 39 chiens atteints et de 38 chiens indemnes âgés de plus de trois ans. Un marqueur microsatellite fortement associé à la CLN ( $\mathrm{p}$ value $=4,1 \times 10^{13}$ ) a été identifié, il est localisé sur le chromosome 9. L'utilisation de 14 marqueurs génétiques supplémentaires, situés sur ce même chromosome, a permis de confirmer l'association. De manière parallèle, 48 chiens américains, appartenant à une grande famille, ont été génotypés pour 315 marqueurs microsatellites. Une liaison génétique forte (LOD score maximal de 9,4) a été mise en évidence dans cette famille entre la CLN et la même portion du chromosome 9 . Douze chiens atteints et 10 chiens sains ont ensuite été génotypés à l'aide de la puce Illumina Infinium Canine SNP170array. Six marqueurs SNP (Single Nucleotide Polymorphism) polymorphes, situés dans la région du chromosome 9 associée et liée à la CLN, ont été identifiés et utilisés pour génotyper les 77 ASTs français. Un haplotype de 678 kilobases, commun à tous les chiens malades, a été mis en évidence à la suite de ce génotypage. En combinant les résultats de l'haplotype des chiens français et de l'analyse de liaison effectuée dans la famille de chiens américains, une région critique de 1039 kilobases, contenant le gène responsable de la CLN, a été identifiée. Cette région critique contenait neuf gènes codant des protéines et un gène codant un petit $A R N$ nucléaire (Abitbol et al. 2010).

\section{Caractérisation des gènes présents dans la région critique}

Afin d'identifier, parmi les neuf gènes candidats présents dans l'intervalle critique, les gènes potentiellement responsables de la CLN une fois mutés, une étude de leur profil d'expression et de leur fonction supposée, a été réalisée. Les données d'expression et de fonction de ces gènes ont été compilées à partir des informations présentes dans les bases de données génomiques humaines et de souris. Les profils d'expression des neuf gènes ont été réalisés, en RT-PCR (Reverse Transcription Polymerase Chain Reaction), à partir d'échantillons de tissus de chien sain. A la suite de ces analyses, trois gènes étaient candidats : ABCA5 (ATP-binding cassette, subfamily A, member 5) en raison de la présence du marqueur microsatellite le plus associé à la CLN dans un de ses introns; ARSG (Arylsulfatase G) et MAP2K6 (Mitogen-activated protein kinase 6) en raison de leur expression dans le système nerveux central chez le chien et de leur rôle possible dans l'homéostasie neuronale. Les exons et les jonctions intron-exon de ces trois gènes ont été séquencés chez quatre chiens atteints de CLN et quatre chiens sains. Deux variations ponctuelles de séquence ont été identifiées entre les chiens sains et les chiens malades : un SNP synonyme (n'entrainant pas de changement d'acide aminé dans la protéine) dans ABCA5 et un SNP non synonyme (entrainant un changement d'acide aminé dans la protéine) dans l'exon 2 du gène ARSG appelé ARSG-SNP (Abitbol et al. 2010)

\section{Identification d'un variant génétique fortement associé à la CLN}

Les 138 chiens atteints de CLN et 181 chiens sains ont été génotypés pour le variant ARSG-SNP. Tous les chiens atteints étaient homozygotes pour l'allèle A du SNP, 50\% (91/181) des chiens

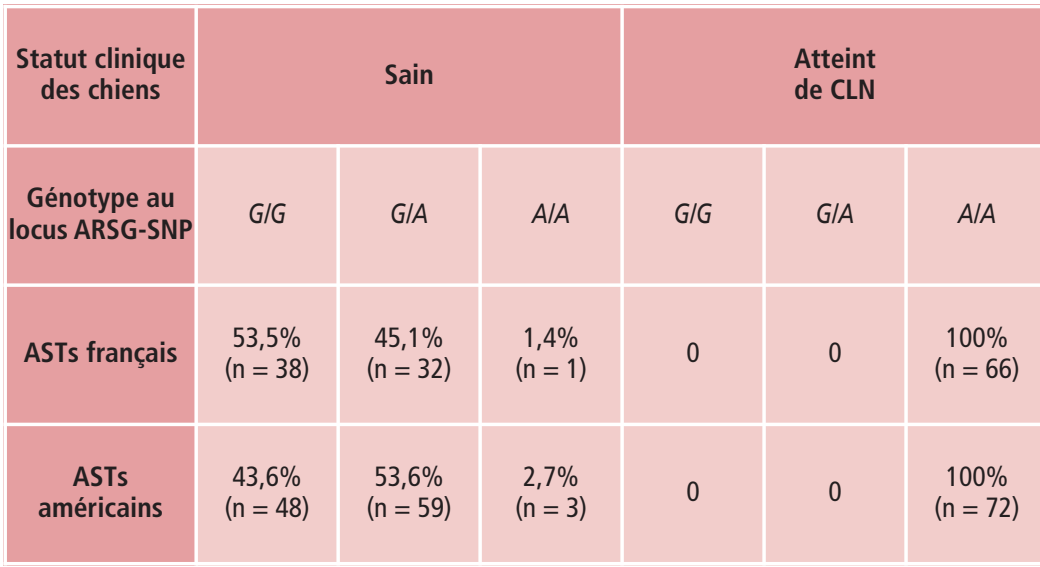

Tableau 1 : Fréquence des génotypes pour le SNP situé dans le gène ARSG, chez des ASTs sains et atteints de cérö̈de-lipofuscinose neuronale (D'après Abitbol et al. 2010). 
sains étaient hétérozygotes $\mathrm{A} / \mathrm{G}$ et $47 \%$ des chiens sains (86/181) étaient homozygotes pour l'allèle G (tableau 1). Le génotypage de 525 chiens appartenant à 54 autres races que l'AST n'a pas permis de mettre en évidence l'allèle A chez ces chiens. Ce variant semblait donc spécifique à la race AST et fortement associé à la CLN. Cependant, quatre chiens appartenant au groupe des chiens sains ont été génotypes A/A pour le SNP. Trois de ces chiens, âgés de neuf ans au moment de l'analyse (juin 2010) n'ont pas pu être examinés par imagerie par résonance magnétique, leur propriétaire n'ayant pas donné leur accord. Le quatrième est mort à l'âge de 13 ans d'une maladie non neurologique. L'analyse histologique de coupes de son cervelet a permis de mettre en évidence une accumulation modérée de céroïde-lipofuscine dans les cellules de Purkinje, différente de celle observée en routine chez les chiens âgés. Cette accumulation fut considérée comme une conséquence subclinique de l'expressivité réduite de la mutation.

Ainsi, l'analyse de la ségrégation du SNP présent dans le gène de l'ARSG, dans de nombreuses races canines, a permis de mettre en évidence son association spécifique à la CLN chez les ASTs (Abitbol et al. 2010).

\section{Caractérisation fonctionnelle du variant associé à la CLN}

Le SNP identifié dans le gène ARSG et spécifiquement associé à la CLN chez les ASTs, provoque un changement d'une arginine en une histidine, en position 99 dans la protéine ARSG. L'ARSG appartient à une famille de sulfatases, conservée chez les métazoaires (Sardiello et al. 2005). Il s'agit d'une enzyme lysosomiale

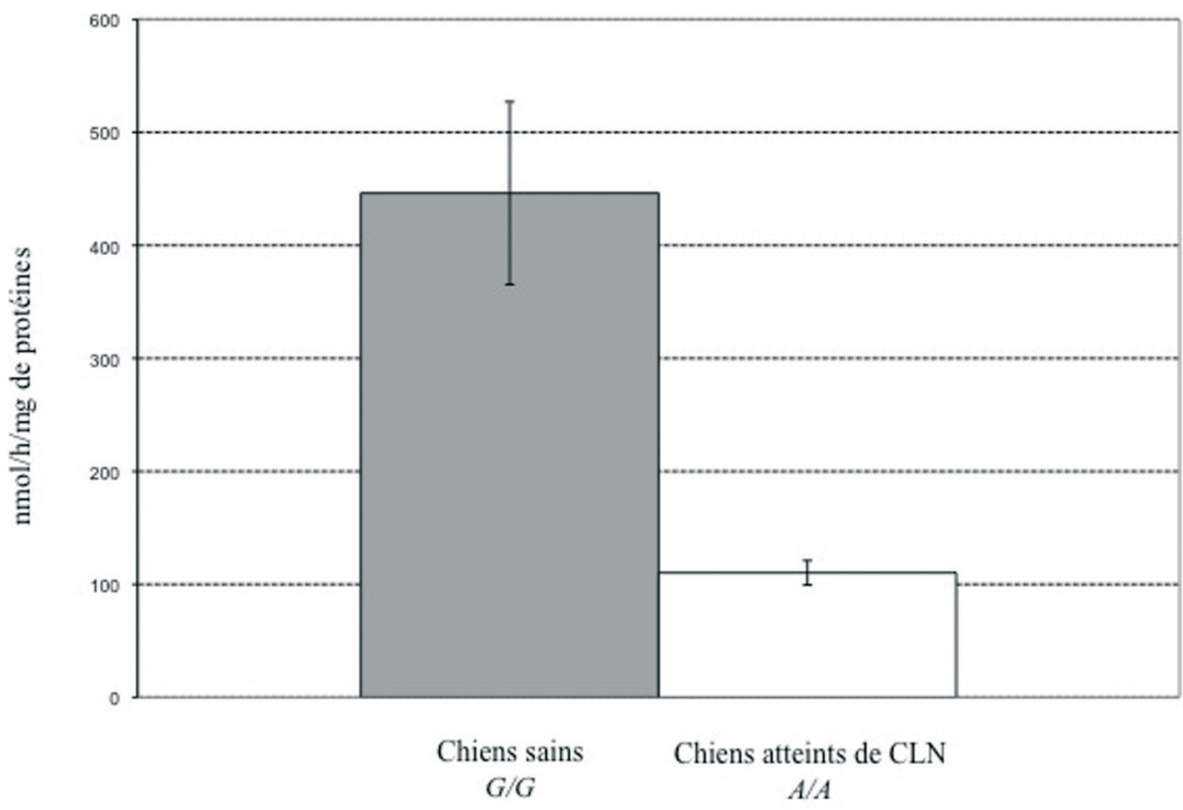

Figure 3 : Activité arylsulfatase $\mathrm{G}$ dans les lymphocytes de cinq chiens American Staffordshire Terrier sains homo zygotes G pour ARSG-SNP et de cinq chiens atteints de céroïde-lipofuscinose neuronale (CLN), homozygotes A pour ARSG-SNP. L'incubation avec le substrat artificiel para-nitrocatécholsulfate a duré une heure. Les barres verticales représentent les erreurs standard à la moyenne (Source : UMR955 INRA-ENVA Génétique Fonctionnelle et Médicale). caractérisée récemment (Frese et al. 2008). Un alignement des séquences protéiques des arylsulfatases humaines fonctionnelles (ARSA à ARSF) avec l'ARSG canine et six arylsulfatases de métazoaires (souris, poule, poisson Taxifugu rubripes, poisson Danio rerio, nématode Caenorhabditis elegans et ascidie Ciona intestinalis) a montré que l'arginine 99 de l'ARSG canine est hautement conservée entre toutes ces espèces et qu'elle est située à proximité du site catalytique de l'enzyme (Ghosh 2005). De plus, l'arginine 99 de l'ARSG canine correspond à l'arginine 84 de l'arylsulfatase A (ARSA) humaine. Deux mutations conduisant à un remplacement de cette arginine 84 ont été décrites chez l'homme. Elles provoquent une baisse de l'activité catalytique de l'ARSA et se traduisent cliniquement par l'apparition d'une maladie neurodégénérative appelée leucodystrophie métachromatique (Kappler et al. 1992 ; Gort et al. 1999; Biffi et al. 2008). Notons enfin que des mutations dans six sulfatases lysosomiales ont été décrites et qu'elles conduisent à des maladies de stockage lysosomiales qui ne sont pas des CLNs (Sardiello et al. 2005 ; Frese et al. 2008).

Les conséquences fonctionnelles du changement d'acide aminé dans l'ARSG des chiens atteints de CLN ont été testées dans un modèle cellulaire, puis in vivo. L'activité de l'ARSG envers un de ses substrats artificiels, le paranitrocatécholsulfate, a été évaluée dans des cellules surexprimant l'ARSG humaine normale et dans des cellules surexprimant l'ARSG humaine portant la mutation canine (une histidine en position 99 au lieu d'une arginine). Dans ce modèle cellulaire, l'activité spécifique de l'ARSG mutée n'a atteint que $18 \%$ de l'activité mesurée chez les cellules surexprimant l'enzyme normale et elle ne différait pas de l'activité enzymatique mesurée dans les cellules témoins qui ne surexprimaient ni l'ARSG normale ni l'ARSG mutée.

Le gène ARSG ayant un profil d'expression ubiquitaire, il a été possible de mesurer l'activité de l'ARSG in vivo chez des ASTs atteints de CLN et chez des témoins sains. L'activité enzymatique de l'ARSG, envers le paranitrocatécholsulfate, a été évaluée dans des lymphocytes de chiens prélevés par ponction sanguine et mis en culture (figure 3). L'activité de l'enzyme, chez les cinq chiens atteints de CLN et homozygotes pour l'allèle muté de ARSG-SNP (allèle A) était réduite de 75\% par rapport à l'activité de l'enzyme chez les cinq chiens sains homozygotes pour l'allèle sauvage de ARSG-SNP (allèle G).

Ainsi, le changement de l'arginine 99 par une histidine, dans l'ARSG canine, conduit à une réduction drastique de l'activité de l'enzyme (Abitbol et al. 2010). 


\section{CONCLUSION : UNE MUTATION DANS LE GÈNE DE L'ARYSULFATASE G, RESPONSABLE DE LA CLN}

L'étude phénotypique de l'ataxie cérébelleuse de l'AST a permis de révéler l'origine de cette maladie, due à une accumulation de céroïde-lipofuscine dans les neurones et à la disparition des cellules de Purkinje, chez les chiens atteints. Les ASTs ataxiques présentent donc une CLN d'apparition tardive. L'étude génétique de la maladie a mis en évidence le mode de transmission autosomique récessif de cette CLN et la mutation causale. Les ASTs atteints de CLN présentent, à l'état homozygote, une mutation dans l'exon 2 du gène ARSG. Cette mutation conduit à un changement d'acide aminé dans la protéine, qui provoque une baisse de 75\% de l'activité arylsulfatase de l'enzyme (Abitbol et al. 2010).
La mise en évidence de cette mutation a permis le développement et la commercialisation d'un test génétique de dépistage de la maladie chez les ASTs (www.antagene.com). Ce test est utilisé par les éleveurs pour déterminer le statut de leurs reproducteurs et adapter les accouplements.

La CLN de l'AST représente le second modèle canin de CLN d'apparition tardive, pour les CLNs humaines adultes (maladie de Kufs), dont l'étiologie moléculaire reste inconnue à ce jour. L'analyse du gène ARSG dans des familles où ségrége une CLN d'origine indéterminée et chez des patients atteints de maladie de Kufs est en cours.

Enfin, le modèle canin AST représente le premier exemple de déficience en sulfatase lysosomiale conduisant à une CLN. Il offre une occasion unique d'établir un lien fonctionnel, encore non élucidé à ce jour, entre l'activité sulfatase lysosomiale et les protéines CLN.

\section{BIBLIOGRAPHIE}

- Abitbol, M., Thibaud, J-L., Olby, N.J., Hitte, C., Puech, J-P., Maurer, M., Pilot-Storck, F., Hédan, B., Dréano, S., Brahimi, S. et al. 2010. A canine Arylsulfatase G (ARSG) mutation leading to a sulfatase deficiency is associated with neuronal ceroid lipofuscinosis. Proc Natl Acad Sci U S A. 107(33):14775-80.

- Bellettato, C.M., Scarpa, M. Pathophysiology of neuropathic lysosomal storage disorders. 2010. J Inherit Metab Dis. 33(4):347-62.

- Berkovic, S.F., Carpenter, S., Andermann, F. Andermann, E., Wolfe, L.S. 1988. Kufs' disease: a critical reappraisal. Brain 111 (Pt 1):27-62.

- Biffi, A., Cesani, M., Fumagalli, F., Del Carro, U., Baldoli, C., Canale, S., Gerevini, S., Amadio, S., Falautano, M., Rovelli, A. et al. 2008. Metachromatic leukodystrophy - mutation analysis provides further evidence of genotype-phenotype correlation. Clin Genet. 74(4):349-57.

- Farias, F.H., Zeng, R., Johnson, G.S., Wininger, F.A., Taylor, J.F., Schnabel, R.D., McKay, S.D., Sanders, D.N., Lohi, H., Seppälä, E.H. et al. 2011. A truncating mutation in ATP13A2 is responsible for adult-onset neuronal ceroid lipofuscinosis in Tibetan terriers. Neurobiol Dis. 2011 Feb 26 [Epub ahead of print].

- Fiske, R.A. \& Storts, R.W. 1988. Neuronal ceroid-lipofuscinosis in Nubian goats. Vet Pathol. 25:171-173.

- Frese, M.A., Schulz, S., Dierks, T. 2008. Arylsulfatase G, a novel lysosomal sulfatase. J Biol Chem. 283:11388-11395.

- Ghosh D. 2005. Three-dimensional structures of sulfatases. Methods Enzymol. 400:273-293.

- Gort, L., Coll, M.J., Chabás, A. 1999. Identification of 12 novel mutations and two new polymorphisms in the arylsulfatase $A$ gene: Haplotype and genotype-phenotype correlation studies in Spanish metachromatic leukodystrophy patients. Hum Mutat. 14:240-248

- Haltia, M. 2006. The neuronal ceroid-lipofuscinoses: from past to present. Biochim Biophys Acta 1762(10):850-856.

- Jalanko, A. \& Braulke, T. 2009. Neuronal ceroid lipofuscinoses. Biochim Biophys Acta 1793(4):697-709.

- Hanzlicek, D., Kathmann, I., Bley, T., Srenk, P., Botteron, C., Gaillard, C., Jaggy, A. 2003. Cerebellar cortical abiotrophy in American Staffordshire terriers: clinical and pathologica description of 3 cases. Schweiz Arch Tierheilkd. 145(8):369-375.

- Kuwamura, M., Nakagawa, M., Nabe, M. Yamate, J., Inoue, M., Satoh, H., Yamato, O. 2009 Neuronal ceroid-lipofuscinosis in a Japanese domestic shorthair cat. J Vet Med Sci. 71(5):665-667.

- Lewandowska, E., Lipczy ska-Łojkowska, W., Modzelewska, J., Wierzba-Bobrowicz, T., Mierzewska, H., Szpak, G.M., Passenik, E., Jachi ska, K. 2009. Kufs' disease: Diagnostic dificulties in the examination of extracerebral biopsies. Folia Neuropathol. 47:259-267.

- Luiro, K., Kopra, O., Blom, T., Gentile, M. Mitchison, H.M., Hovatta, I., Tornquist, K., Jalanko, A. 2006. Batten disease (JNCL) is linked to disturbances in mitochondrial, cytoskeletal, and synaptic compartments. J Neurosci Res. 84(5):1124-1138.

- Lyly, A., von Schantz, C., Heine, C., Schmiedt, M.L., Sipila, T., Jalanko, A., Kyttala, A. 2009. Novel interactions of CLN5 support molecular networking between Neuronal Ceroid Lipofuscinosis proteins. BMC Cell Biol. 10: 83.
- Olby, N., Blot, S., Thibaud, J-L., Phillips, J. O'Brien, D.P., Burr, J., Berg, J., Brown, T., Breen, M. 2004. Cerebellar cortical degeneration in adult American Staffordshire Terriers. J Vet Intern Med. 18(2):201-208.

- Saja, S., Buff, H., Smith, A.C., Williams, T.S., Korey, C.A. 2010. Identifying cellular pathways modulated by Drosophila palmitoyl-protein thioesterase 1 function. Neurobiol Dis. 40(1):135-145.

- Sardiello, M., Annunziata, I., Roma, G. Ballabio, A. 2005. Sulfatases and sulfatasse modifying factors: An exclusive and promiscuous relationship. Hum Mol Genet. 14:3203-3217.

- Seehafer, S.S. \& Pearce, D.A. 2009. Spectral properties and mechanisms that underlie autofluorescent accumulations in Batten disease. Biochem Biophys Res Commun. 382(2): 247-251.

- Sisó, S., Navarro, C., Hanzlícek, D., Vandevelde, M. 2004. Adult-onset thalamocerebellar degeneration in dogs associated to neuronal storage of ceroid lipopigment. Acta Neuropathol. 108: 386-392.

- von Schantz, C., Saharinen, J., Kopra, O. Cooper, J.D., Gentile, M., Hovatta, I., Peltonen, L., Jalanko, A. 2008. Brain gene expression profiles of $\mathrm{Cln} 1$ and $\mathrm{Cln} 5$ deficient mice unravels common molecular pathways underlying neuronal degeneration in NCL diseases. BMC Genomics 9:146.

- Zhao, L. \& Ackerman, S.L. 2006. Endoplasmic reticulum stress in health and disease. Curr Opin Cell Biol. 18:444-452. 